self-reliance. Turn their thoughts towards recovery, not hypochondriasis. Make them understand that you will assist, not sympathize with every imaginary ache and pain. Trust them and encourage them in their effort towards recovery; even "if they fall by the wayside," try it again. Do not encourage morbidness, for we all know the craving for sympathy that invariably persists even when partially convalescent. Avoid drugs, especially anodynes, hypnotics and opiates, Treat upon general principles, no matter if it is oldfashioned. It is no sign of mental decrepitude if our patient retains her ovaries intact or trachelorrhaphy has never been performed. Again repeating that, while admiring the skill and general worth of our brethren of special faith, they must always remember that general medicine is the sine qua non of success.

\section{OBSERVATIONS ON YELLOW FEVER, WITH SPECIAL REFERENCE TO DIAGNOSIS, PROGNOSIS AND TREATMENT.}

Reati before the State Medical Association of Florita, 1888. BY JOHN P. WALL, M.D., OF TAMPA, FLA.

The following remarks on yellow fever are based on clinical observations during the epidemic of that disease last fall in Tampa.

The etiology of this fever is but little, if any better understood than its pathology; and in these two points are to be found the individual characteristics, so to speak, of the disease. The idea of yellow fever being only a more malignant grade of malarial fever can only be entertained by very superficial observers, or those who have never passed through an epidemic of this fever. Notwithstanding this broad assertion it is not by any means easy, nor in fact always possible, to determine whether any single case is yellow fever or not ; and this is especially the case at the inception of an epidemic. To arrive at a conclusion on this point quite a number of circumstances in connection with the case, apart from the symptoms, have to be taken into consideration; and then, reasoning by a process of exclusion, we may be able to approximate, at least, the probable truth. The recent history of the patient as to being exposed to infection in another place, on board ship or otherwise, and whether or not the patient has had a previous attack of this specific fever, are circumstances of the first importance to be taken into consideration. Though the patient may be from an infected place or have been otherwise exposed to the infection, yet if it can be positively established that he has had yellow fever, the probability of his having a second attack is so unlikely and remote as to warrant the exclusion of the yellow fever hypothesis in arriving at a diagnosis.
But where the patient has recently been exposed to infection and possesses no immunity acquired by a previous attack from this specific fever-in other words, is not acclimated-then, in such a case, there is a probability of its being yellow fever, and advantage should be taken of the doubt and the case managed as a case of yellow fever without waiting for a positive diagnosis. The management of such a case consists in the complete and thorough isolation of patient and attendants and, on the termination of the case, the prompt institution of measures for efficient disinfection of every thing, even to premises, that might have been contaminated.

In its symptomatology yellow fever presents no well-marked indications of its true character in the beginning; nor, for that matter, at any time in mild cases, during its progress. Its attack is generally ushered in by a chill or chilly sensation, as in most other fevers and the pyrexiæ. With the rise of fever there is generally intense frontal headache, with pain in the small of the back and extremities, especially the legs. A feeling of tiredness in the legs, with a sense of general prostration, is usually experienced. Nausea from the beginning is not an uncommon symptom, though not always present. Incessant nausea with much retching or vomiting, during the first exacerbation of fever, indicates the case to be of a severe type. The tongue is generally coated with a white or whitish fur, is sometimes enlarged, and almost invariably presents red edges on the sides. The temperature for the first three days may range in different cases from $102^{\circ}$ to $106^{\circ} \mathrm{F}$., with a slight morning remission in the majority of cases. The pulse for the first three days may range in different cases from go to I ro per minute, though not so frequently above as below roo beats per minute.

The skin is generally burning hot, rarely dry, and not unfrequently bathed in profuse perspiration. In many cases, after the fever sets in, the extremities remain cool, while the body heat is intense and pungent. And it is in this class of cases where mustard pediluvia or mustard sitz baths have been found so useful and beneficial in restoring the eqilibrium of the circulation, as to render the mustard bath an almost universal remedy in the beginning of treatment. In severe cases albumin will be found in the urine on the third day, as a rule. Its presence is always a grave symptom, but as it is rarely-never in my observations-to be detected before the third day, it is impossible to determine up to that time whether the case is to be of a mild or grave type. In all cases where albumin is not present in the urine on the third day or at the expiration of seventy-two hours from the inception of the fever, the case will, as a rule, prove to be of a mild type, and the patient and friends can be assured of a speedy recovery if great prudence in eating and drinking is exercised for two or three days more. 
And just here it is well to distinctly emphasize the fact that it is the acute parenchymatous nephritis, as indicated by the presence of albumin in the urine on the third day, which is the most marked characteristic of the graver type of this fever, and without which the icteric hue of the conjunctivæ and skin is rarely, if ever, developed. But it must be borne in mind that by no means do all cases of this fever, as it presents itself in this country, have albumin in the urine. And it is the presence of albumin in the urine on the third day, subseqently followed by the icteric hue of the conjunctiva and skin, by which this fever can be positively diagnosticated. These two symptoms-albumin in the urine and the icteric hueat the proper time and in proper sequence, render the diagnosis of yellow fever as positive, from a scientific point of view, as it is possible to diagnosticate any other disease by physical symptoms.

The later writers and authors speak of albumin appearing in the urine in yellow fever, but fail to indicate at what time or stage of the disease the albumin is to be found; and they also fail to say that it is not present in all cases-two facts of the first importance. In the London Lancet of March I 7, I 888, Dr. Robert Lawson, Inspector-General of the British Army, who had served in Jamaica, says: "On the evening of the third day, or morning of the fourth, the urine generally presents traces of albumin," thus confirming my observations as to the time when the presence of albumin in the trine may be expected. He fails to state, however, that albumin is not present in all cases of the fever, a fact of the highest importance in eliminating much of the confusion in regard to the diagnosis. In mild cases, where no albumin is found in the urine on the third day, the fever will terminate on the expiration of seventy-two hours, sometimes earlier, not to return, and the patient will progress to rapid convalescence without further trouble.

Yellow fever is generally described by authors as a fever of one paroxysm of seventy-two hours' duration, followed by a stage of apyretic calm of variable length, from one to twelve hours, and this is succeeded by the recurrence of fever of more or less intensity, accompanied with nausea and vomiting of white glairy mucus at first, then flecked with dark specks and shreds, which have been compared to bees' or butterflies' wings, finally terminating in the coffee-grounds-like black vomit. Now, as a rule, all of this is true of typically bad cases, but this secondary fever and nausea are very rarely to be met with in mild cases devoid of albumin in the urine on the third day. And in all cases in which there is no nephritic complication, as indicated by the absence of albumin in the arine, yellow fever ends on the third day, or perhaps earlier, convalescence practically sets in and recovery is soon complete.

Thus it will be seen that practically there are two types or grades of this fever to be met with in an epidemic-one whose main feature is an acute parenchymatous nephritic complication developed by the third day; and the other which, having no such complication, ends with the first paroxysm of the fever and almost invariably terminates in speedy recovery, the patient being up and about as early as the fifth or sixth day. Not so, however, where albumin is detected in the urine on the third day; for while, in this class of cases, the high fever of the first three days may subside, and the pulse rate return to the normal, or even fall much lower, and the temperature be anywhere from $97^{\circ}$ to $104^{\circ} \mathrm{F}$., the patient still continues in a most critical condition, and not unfrequently so remains for an indefinite number of days. In some instances the temperature and pulse are normal, and so remain after the subsidence of the first paroxysm of the fever; but there are plenty of indications, and especially the albumin in the urine, to show the serious condition of the patient. It is doubtless this class of cases to which relapses are attributed by a certain class of physicians who are always trying to explain away why they failed to cure all their cases of yellow fever.

The amount of albumin in the urine varies in different cases, ranging from 5 to 75 per cent. of volume of the total amount; and, as a rule, the greater the amount of albumin, the more serious and less hopeful may be considered the prognosis. In these nephritic cases the eyes begin to show the icteric hue on the fifth day, and on the sixth or seventh day the skin begins to turn yellow. In rapidly fatal cases, terminating as early as the fourth or fifth day, the yellow hue appears earlier and is always well-marked after death. The yellow color which is characteristic of this fever in its worse and most fatal type, and has given the fever its name, is very rarely indeed, if ever, present in cases unaccompanied by the nephritic complication. This is repeated because it is a most important fact to remember.

Hæmorrhages from mouth and nose are not infrequent in the severe grade of cases having the nephritic complication. The urine may be tinged with blood, imparting to the coagulated albumin a dark color, but hæmorrhage from the urinary organs is seldom profuse; and as these hæmorrhages never occur in the paroxysm of the fever of the first three days, and only in cases with the nephritic complication, there should never be any trouble in differentiating yellow fever from hæmorrhagic malarial fever. The eyes become bloodshotten in no inconsiderable number of cases. Epistaxis and bleeding from the gums are the more common forms of hæmorrhage met with in this fever, and the nose-bleed is often alarming. Among females passed puberty, more or less uterine hæmorrhage is almost certain to occur during the course of the fever, making its appearance 
from the third to the fifth day. Even in mild and skin, establish the diagnosis of the fever becases this uterine hæmorrhage is rarely absent- yond all doubt. But as before pointed out these and by mild cases are understood those without two symptoms are absent in mild cases-those the nephritic complication.

The icteric hue of conjunctivæ and skin has very much the appearance of ordinary jaundice, though it is hardly likely that it is produced by the same cause-retention of bile in the bloodfor the reason that bile is not very frequently found in the urine, as is universally the case in ordinary jaundice. One of the tests for bile in the urine is to add albumin and precipitate it with heat and an acid, when the coagulated albumin will assume a greenish color, imparted by the presence of biliverdin. In cases with the nephritic complication the albumin is already present in the urine, but it is only occasionally that the precipitated albumin assumes the greenish color of the biliverdin. From the time the yellowness of the skin becomes fully developed until it begins to fade, the capillary circulation is extremely sluggish; so much so, in fact, that firm pressure on the chest with the open and separated fingers leaves their marks on the surface for a number of seconds if not a full minute. Judging from the tendency to hæmorrhage and the capillary stasis which are such prominent features in severe cases of this fever, and taking into consideration the $a b$. sence of bile in the urine in the large majority of cases, the conclusion is highly probable, if not positive, that this yellowness must be mainly dependent on pigmentation of the eyes and skin by the coloring matter of the blood, instead of being occasioned by the retention of bile in the blood-as in ordinary jaundice-as is generally supposed and believed.

Suppression of urine, or a very scant secretion more or less tinged with blood, only occurs in the third stage of the fever; rarely, if ever, during the paroxysm of the first three days. And it is only in this graver type of cases, with the nephritic complication, that suppression of urine takes place, to be followed by spasmodic twitchings, stupor, convulsions and death. These muscular twitchings are especially noticeable about the face. These nervous phenomena are supposed to be the effects of uræmic poisoning as a result of nonexcretion of urea by the kidneys and its retention in the blood. But be that as it may, their occurrence is almost a certain prelude to a fatal termination, and in a prognostic sense is as bad, if not worse, than the black vomit. Hiccough is never met with in the first fever, $i . e$. , before the fourth or fifth day, and is a symptom of the most unfavorable import.

The injected and watery eyes, swollen face and anxious countenance so generally depicted by writers on this fever, may be noticed in a large number of cases, probably the majority. The detection of albumin in the urine on the third day of the fever and the subsequent icteric hue of eyes unattended with the nephritic complication-and hence the frequent disputes and contentions as to diagnosis in the beginning of an epidemic. The duration of the paroxysm of the fever for only seventy-two hours, or even perhaps a shorter time, and its abrupt termination without the use of quinine, should excite, however, grave suspicion of its character ; and this suspicion will be greatly strengthened if the chill come on in the night, and especially in the latter half of the night. Dengue is the only other fever of our climate which may be ushered in by a chill at night, but its symptoms are too well marked-besides being a proverbially non-fatal disease-to admit of its being mistaken for yellow fever, or, vice versa, yellow fever being mistaken for dengue, by any one but the merest tyro in the profession-especially after a few days study and observation.

This brevity of the first paroxysm of yellow fever in mild cases, the subsidence of the fever without the use of quinine, the severe frontal headache, with pain in the back and extremities, and the absence of a second paroxysm of fever, taken with the appearance of the eruption on the extremities associated with dengue, will rarely fail to indicate to the experienced physician the true nature of the case. Many yellow fever patients emit a peculiar odor which the experienced physician has no difficulty in recognizing, though he may not be able to describe it by comparing it to any similar smell.

The matter of early diagnosis is a most important one in our Southern cities and towns as it is generally conceded, and my own experience tends to confirm this view, that if the first case (or cases) is recognized and proper measures of isolation and disinfection are instituted an epidemic may be prevented, especially in places whose atmosphere is not vitiated with miasmatic exhalations from accumulated filth. Hence the importance of ever being on the alert and carefully investigating any and every case of fever that comes under observation during the season when the fever usually prevails-the summer and fall of the year.

Daily observations should be made for the detection of albumin in the urine from the first to the fourth day, and if its presence is denoted on the third day the diagnosis is probable, and if on the sixth or seventh day there appears the icteric hue of conjunctiva and skin the diagnosis is positive. If the case is mild, however, but with the severe frontal headache and other symptoms already detailed, and abruptly terminates on the expiration of the third day, though no albumin may have been found in the urine, the diagnosis of yellow fever is only problematic, but the benefit of the doubt should be given to the community and all necessary precautions taken to prevent the increase and spread 
of the infection. Of course the previous history of the patient, as to the probability of his having been exposed to the infection, and as to his ever before having had yellow fever, should be duly investigated.

As to treatment little need be said, as, unfortunately, so far no specific treatment has been found. The mild cases of the fever-those destitute of the nephritic complication-will, as a rule, get well spontaneously, with or without treatment, and it is probably because of the failure to recognize the two types of this fever pointed out in this paper that so many different remedies have been vaunted and then laid aside, and so much been said of different epidemics differing in their phases, and of remedies effective in some epidemics and futile or useless is others. The main indications are to restore the equilibrium of the circulation by mustard pediluvia or baths, evacuate the bowels with some efficient though mild purgative, and control the febrile excitement by the use of antipyretics-antipyrin and antifebrin. So far as my experience goes mercurials do not appear to possess any special advantage, except possibly that the stomach is not so likely to reject them as it often does more bulky drugs. Doubtless the common, though possibly erroneous belief that yellow fever is only an intensified form of bilious fever, and that the icteric hue is dependent on the same cause as that producing jaundice, and hence the fault laid to the liver, has always attached more importance to the use of mercury in the shape of calomel in this disease than experience justifies. Castor oil, or the more common salines, will be found about as good evacuants of the prime vice as anything else. Quinine is a useless drug in yellow fever unless there is some evidence of a malarial complication. It may be advantageously used during convalesence, but it is perfectly val ueless in controlling the disease. Administered in mild cases, which would naturally recover, quinine often has received the credit of having cut short the fever as a post hoc propter hoc conclusion. The refraining from its use, even in mild cases, is advantageous in a diagnostic point of view, for if the fever spontaneously terminates by the end of seventy-two hours, or earlier, the presumption is against it having been of a malarial type. Antipyrin in Io grain doses every three hours appears to have a very beneficial effect in reducing temperature, promoting diaphoresis, and relieving headache and other pains. Its advantages over antifebrin are its analgesic properties and greater solubility which renders its administration by enema in 20 or 30 grain doses almost as efficient as when administered per os. And this method of administration sometimes becomes necessary on account of nausea and excessive irritability of the stomach. The use of opiates is always hazardous, unless there was some way of determining anterior to the third day that no nephritic complication would be developed, as their tendency is to induce suppression of urine. Of course in mild cases in which there is no albumin in the urine it is not certain that their use is hurtful, but the difficulty as to their use arises from our inability to determine this very important point as to the nephritic complication; and hence the safe rule is to eschew the use of opiates entirely in the treatment. An occasional exception to this rule may be made in cases presenting other complications and where the kidneys are not, or only slightly, affected.

As to food and drink, the less taken the better, so as to keep the stomach as quiet as possible. Cold drinks are decidedly injurious in the great majority of cases. Hot drinks are borne much better, and hence, doubtless, the common practice of administering lemon leaf and other teas. Possibly the benefit derived from these hot drinks is dependent on their constringing effect on the capillaries of the mucous coat of the stomach, on the same principle that injections of hot water are used in gynecology. For nourishment a tablespoonful of equal parts of milk and lime-water may be given every two hours, though if the patient is not naturally feeble "Tannerism," or starvation, may be practiced.

In the graver type of cases with the nephritic trouble, no line of treatment after the third day, promises any certainty of success. So long as the quantity of albumin in the urine remains comparatively small and the kidneys continue to act tolerably freely there is encouraging hope of the patient "pulling through," though there is no means of telling when or how soon the action of the kidneys will become fatally impeded or suppressed, and the case terminate in stupor, convulsions and death with or without black vomit toward the end. It is in this class of cases that we are constrained to recognize the impotency of our art and our utter helplessness in the presence of this dread disease. In this latter stage the tincture of digitalis in ro to 15 drop doses every two or three hours may prove of some advantage as a heart tonic and diuretic. Of course, alcoholic preparations are indicated to sustain the flagging vital powers; but, unfortunately, like a great many other remedies, disappointment is only too frequently the result of their use. In the mild cases without the nephritic trouble alcohol is rarely indicated, it may be occasionally indicated in the nephritic cases where the quantity of albumin is small and the kidneys act fairly well, while in the worst grade of cases, it, like everything else in the way of remedies, is likely to prove of little avail.

No remedies proved specially useful in relieving nausea and hiccoughs. Cocaine was tried without benefit. Carbolic acid in small doses proved a failure. In fact nothing but as complete abstention as possible from food and drinks appeared to do any good. 
NoTE.-These observations are based upon i 30 the man may have no evidences of urinary obcases that I have treated myself, in which the struction. And, on the other hand, the finger urine was tested, besides seeing a large number in may reveal to your sense of touch no increase in consultation where the urine was examined also. the size of the gland; and yet there may exist Dr. J.Y. Porter, who was here during the epidemic marked prostatic obstruction. There may be new and had charge of the Fever Hospital and the growths, tumors, true myomata which can be Government relief measures, admits that his ob- only felt by the finger in the bladder. The enservations tally in the main with mine.

THE FORMATION OF AN ARTIFICIAL URETHRA FOR PROSTATIC HYPERTROPHY.

Extempore Remarks bcfore the Medical Society of Virginia, Hednesday Evening, October' 24, 1888 .

BY HUNTER MCGUIRE, M.D.,

EX-PRESIDENT OF THE MEDICAL SOCIETY OF VIRGINIA; SLRGEON TO ST. LCKE'S HOSPITAL, RICHMOND, VA.

After relating a case in which members of the Society were personally interested, Dr. McGuire said that this is the way that men usually die (by surgical kidney) who have serious obstruction to the passage of urine. In both sexes, and at all ages, mechanical obstruction to the passage of urine from the kidney ends in cystitis, ureteritis, pyelitis and pyelo-nephrosis. Obstruction may be due to stricture, enlarged prostate, tumor, stone, etc. It ends sooner or later in surgical kidney.

A very common cause for this is an enlarged prostate. This comes on after the age of 55 years. As far as Dr. McGuire's experience goes, if a man escapes this trouble until he is $6_{3}$, he is not apt to have it. We all know how common it is in old men-so common that he sometimes wonders that it was not included in that wonderful description of old age found in the last chapter of Ecclesiastes. Possibly senile hypertrophy did not belong to that period; but is one of the results of so-called modern civilization. We don't know. We only know that hypertrophy of this gland is, with rare exceptions, peculiar to manl. The prostate is a curious body; it is made up of fibrous, glandular and muscular tissue. It would be as well, if not better, to call it the prostatic muscle, than to call it the prostatic gland. It supports the bladder, which, otherwise, is so lightly and so feebly held in its position. The erect posture which man assumes puts a great strain on this body. Indeed, this might be used as an argument that it was intended for man to go on all fours, like the quadrupeds.

When deterioration of all the tissues beginsthat is, about 55 years of age or more-this structure may commence to get bigger; it may enlarge toward the bowel, and do little or no harm. But if its growth encroaches in any way on the lumen of the urethra, then trouble begins. Put your finger into the patient's rectum, and you may find the prostate greatly enlarged; and yet largement may be either centric or eccentric. You cannot reckon the extent of obstruction by the mere size of the gland felt through the rectum. All this depends upon the direction of the growth.

Let it encroach, even slightly, upon the urethral canal, and lessen its size, and there soon follows irritability of the bladder, retention of urine, cystitis; and if this goes on, pyelitis and pyelo-nephrosis.

Dr. McGuire saw before him, as he was making these remarks, which he certainly did not intend to make, and for which he was entirely unprepared, many gray headed men who are listening to him with great attention. Some of you "old Fellows" may already know that your prostates are getting larger than they used to be; that it takes you a little longer to make water now; that it is slow to start; that the stream is not sent as far from your body, but drops down between your legs, which you keep wide apart to prevent your trowsers from being splashed. He advises you to try to empty the bladder every time you urinate, and not leave in it some residual urine which will decompose, just as it would do in a dirty chamber-pot, and afterwards set up in your bladder irritation and inflammation. Take care also to keep your body, and especially your feet, dry and warm. Don't sit on a cold stone or a wet saddle. Keep your bowels open, and let whisky alone. If the enlargement goes on increasing, and the calibre of the urethra is more and more diminished, more prominent and urgent symptons are presented. Sudden retention of urine may occur, requiring the use of the catheter; or (and this is just as frequent) the patient will tell you that he cannot hold his water; that he has incontinence of urine. This condition generally means that his bladder is distended with urine, and overflowing; and the catheter is required here to relieve the over-distension. It would be out of place for him to talk about antiseptics and germs now. But it is not out of place for him to beg you to keep your catheter clean. A dirty catheter is a very dangerous instrument.

After a longer or shorter time, this enlarged prostate forms a dam at the outlet of the bladder, and only the water above the level of the dam escapes during micturition. Some urine, which is called "residual urine," is always left in the bladder. This decomposes, becomes ammoniacal, is an irritant and sets up cystitis. The poor sufferer strains violently to make water. This tenesmus of the bladder provoked by the obstruction, injures the vesical end of the ureters. These 